\title{
Apoptosis induction of human prostate carcinoma cells by cordycepin through reactive oxygen species-mediated mitochondrial death pathway
}

\author{
HYE HYEON LEE ${ }^{1,2}$, CHEOL PARK ${ }^{3,4}$, JIN-WOO JEONG $^{3,4}$, MIN JEONG KIM ${ }^{1,2}$, \\ MIN JEONG SEO ${ }^{2}$, BYOUNG WON KANG ${ }^{2}$, JEONG UCK PARK ${ }^{2}$, GI-YOUNG KIM ${ }^{5}$, \\ BYUNG TAE $\mathrm{CHOI}^{6}$, YUNG HYUN $\mathrm{CHOI}^{3,4,7}$ and YONG KEE JEONG ${ }^{1,2}$
}

${ }^{1}$ Department of Biotechnology, ${ }^{2}$ Medi-Farm Industrialization Research Center, Dong-A University, Busan 604-714;
${ }^{3}$ Department of Biochemistry, Dongeui University College of Oriental Medicine, Busan 614-052;
${ }^{4}$ Anti-Aging Research Center and Blue-Bio Industry RIC, Busan 614-714; ${ }^{5}$ Laboratory of Immunobiology,
Department of Marine Life Sciences, Jeju National University, Jeju 690-756; ${ }^{6}$ Division of Meridian and
Structural Medicine, School of Korean Medicine, Pusan National University, Gyeongnam 626-870;
${ }^{7}$ Department of Biomaterial Control (BK21 program), Dongeui University, Busan 614-714, Republic of Korea

Received October 22, 2012; Accepted December 7, 2012

DOI: $10.3892 /$ ijo.2013.1762

\begin{abstract}
Cordycepin is the main functional component of Cordyceps militaris, which has been widely used in oriental traditional medicine. This compound has been shown to possess many pharmacological properties, such as enhancing the body's immune function, and anti-inflammatory, anti-aging and anticancer effects. In the present study, we investigated the apoptotic effects of cordycepin in human prostate carcinoma cells. We found that treatment with cordycepin significantly inhibited cell growth by inducing apoptosis in PC-3 cells. Apoptosis induction of PC-3 cells by cordycepin showed correlation with proteolytic activation of caspase- 3 and -9 , but not caspase- 8 , and concomitant degradation of poly (ADP-ribose) polymerases, collapse of the mitochondrial membrane potential (MMP). In addition, cordycepin treatment resulted in an increase of the $\mathrm{Bax} / \mathrm{Bcl}-2$ (or Bcl-xL) ratio, downregulation of inhibitor of apoptosis protein (IAP) family members, Bax conformational changes, and release of cytochrome $c$ from the mitochondria to the cytosol. The cordycepin-induced apoptosis was also associated with the generation of intracellular reactive oxygen species (ROS). However, the quenching of ROS generation with antioxidant $\mathrm{N}$-acetyl-L-cysteine conferred significant
\end{abstract}

Correspondence to: Dr Yong Kee Jeong, Department of Biotechnology, Dong-A University, Busan 604-714, Republic of Korea E-mail:rmflsq112@naver.com

Dr Yung Hyun Choi, Department of Biochemistry, Dongeui University College of Oriental Medicine, San 45, Yangjung-dong Busanjin-gu, Busan 614-052, Republic of Korea

E-mail: choiyh@deu.ac.kr

Key words: cordycepin, PC-3 cells, apoptosis, reactive oxygen species protection against cordycepin-elicited ROS generation, disruption of the MMP, modulation of Bcl-2 and IAP family proteins, caspase- 3 and -9 activation and apoptosis. This indicates that the cellular ROS generation plays a pivotal role in the initiation of cordycepin-triggered apoptotic death. Collectively, our findings suggest that cordycepin is a potent inducer of apoptosis of prostate cancer cells via a mitochondrial-mediated intrinsic pathway and that this agent may be of value in the development of a potential therapeutic candidate for both the prevention and treatment of cancer.

\section{Introduction}

Prostate cancer is the most frequently diagnosed malignancy and the second most common cause of cancer death in men. It occurs predominantly in persons over 50 years of age (1), typically progressing at a slow rate (2). Prostate cancer in elderly males accounts for $33 \%$ of all newly diagnosed malignancies among men in the United States (3). Moreover, the number of patients with prostate cancer is increasing in Asia $(4,5)$. Therefore, the exploration and development of novel and more effective antitumor agents for patients with prostate cancer are urgently needed.

Apoptosis is a highly regulated process of programmed cell death that plays an important role in the maintenance of cellular homeostasis. Disruption of this process represents a major contributing factor in the pathology of cancer. Thus, apoptosis activation has been considered a good target in cancer therapies $(6,7)$. In general, apoptosis is regulated by pro-apoptotic and anti-apoptotic gene products, such as the Bcl-2 and inhibitor of apoptosis protein (IAP) family members, and executed through caspases and cysteine-aspartic proteases, chiefly via two major and inter-related pathways (i.e., the mitochondriadependent 'intrinsic' cytochrome $c /$ caspase-9 pathway and the death receptor-mediated 'extrinsic' caspase- 8 pathway) $(8,9)$. 
Caspase activation further leads to protein cleavage resulting in DNA fragmentation, chromatin condensation and cell shrinkage. Additionally, reactive oxygen species (ROS) play a key role in mitochondria-mediated apoptosis. Mitochondria are the prime source of ROS, which are byproducts of aerobic respiration $(10,11)$. High levels of ROS in mitochondria can result in free radical attack of membrane phospholipids and cause mitochondrial membrane depolarization. This is an irreversible step, which is associated with the release of mitochondrial factors including cytochrome $c$, triggering caspase cascades $(12,13,14)$. Therefore, ROS plays an important role in mitochondria-mediated apoptotic pathway.

Cordycepin, 3'-deoxyadenosine, is a major functional component in the Cordyceps militaris fungus (Fig. 1) $(15,16)$. Due to the absence of oxygen in the 30-position of its ribose moiety, the incorporation of cordycepin during RNA synthesis will result in termination of chain elongation. This activity has been well described in vitro with purified RNA polymerases and poly(A) polymerases from a number of organisms, including yeast and mammals $(17,18)$. Cordycepin has also demonstrated various properties, such as antitumor $(18,19,20)$, anti-fungal (21), anti-bacterial (22) and anti-inflammatory effects $(23,24)$. Indeed, for centuries, Cordyceps militaris has been a widely administered traditional Chinese medicine, with cordycepin believed to be one of the bioactive components mediating its beneficial effects $(16,25)$. While well known as a therapeutic agent due to its unique properties, the molecular mechanisms underlying the anticancer effects of cordycepin are not yet completely understood.

The purpose of this study was to evaluate the role of mitochondria in apoptosis induced by cordycepin, using human prostate carcinoma cells. We examined whether ROS were critical mediators of cordycepin-induced PC-3 cell death, and we determined the sequence of events leading to the activation of downstream caspases and apoptosis. The study furnishes evidence that cordycepin elicits ROS, which in turn triggers a decrease in mitochondria membrane potential (MMP), consequently leading to caspase activation.

\section{Materials and methods}

Reagents and antibodies. Cordycepin (MW, 251.2; product no. C3394), 4,6-diamidino-2-phenylindole (DAPI), dimethyl sulfoxide(DMSO),N-acetyl-L-cysteine(NAC),3-(4,5-dimethyl2-thiazolyl)-2,5-diphenyl-2H-tetrazolium bromide (MTT), 5,5',6,6'-tetrachloro-1,1',3,3'-tetraethyl-imidacarbocyanine iodide (JC-1) and propidium iodide (PI) were purchased from the Sigma-Aldrich Chemical Co. (St. Louis, MO). Fetal bovine serum (FBS) and caspase activity assay kits were obtained from Gibco-BRL (Grand Island,NY) and R\&D Systems (Minneapolis, $\mathrm{MN})$, respectively. The DNA staining kit (CycleTEST $^{\mathrm{TM}}$ Plus Kit) and enhanced chemiluminescence (ECL) kit were purchased from Becton-Dickinson (San Jose, CA) and Amersham Co. (Arlington Heights, IL), respectively. Antibodies specific for XIAP, cIAP-1, cIAP-2, Bcl-2, Bax, Bcl-xL, caspase-3, -8 and -9, and poly(ADP-ribose)polymerases (PARP) were obtained from Santa Cruz Biotechnology (Santa Cruz, CA). Anti-cytochrome $c$ and actin antibodies were purchased from Cell Signaling (Beverly, MA) and Sigma-Aldrich Chemical Co., respectively. The peroxidase-labeled donkey anti-rabbit immunoglobulin and peroxidase-labeled sheep anti-mouse immunoglobulin were purchased from Amersham Co.

Cell lines, cell culture and MTT assay. Human prostate cancer cell lines (PC-3, DU145 and LNCaP) were obtained from the American Type Culture Collection (Rockville, MD). The culture medium used throughout the experiments was RPMI-1640 medium (Gibco-BRL), containing 10\% FBS, 2 mM L-glutamine, and $100 \mathrm{U} / \mathrm{ml}$ penicillin and streptomycin. Cells were cultured at $37^{\circ} \mathrm{C}$ in a humidified chamber containing $5 \% \mathrm{CO}_{2}$. For the cell viability assay, cells were seeded in 6-well plates and treated with various concentrations of cordycepin for $24 \mathrm{~h}$. After treatments, MTT working solution was added to 6 -well culture plates and incubated continuously at $37^{\circ} \mathrm{C}$ for $2 \mathrm{~h}$. The culture supernatant was removed from the wells and DMSO was added to dissolve the formazan crystals. The absorbance of each well was measured at $540 \mathrm{~nm}$ with an ELISA reader (Molecular Devices, Sunnyvale, CA).

Flow cytometry analysis. After treatment with cordycepin, the cells were collected, washed with cold phosphate-buffered saline (PBS) and fixed in $75 \%$ ethanol at $4^{\circ} \mathrm{C}$ for $30 \mathrm{~min}$. Prior to analysis, the cells were washed once again with PBS, suspended in a cold PI solution containing $100 \mu \mathrm{g} / \mathrm{ml}$ RNase A, $50 \mu \mathrm{g} / \mathrm{ml} \mathrm{PI}, 0.1 \%(\mathrm{w} / \mathrm{v})$ sodium citrate and $0.1 \%$ (v/v) NP-40, and further incubated on ice for $30 \mathrm{~min}$ in the dark. Flow cytometry analyses were carried out using a flow cytometer (FACSCalibur; Becton-Dickinson). Cell-Quest software was used to determine the relative DNA content based on the presence of red fluorescence. The sub-G1 population was calculated to estimate the apoptotic cell population (26).

DNA fragmentation assay. Cells were lysed in $100 \mu \mathrm{l}$ of $10 \mathrm{mM}$ Tris- $\mathrm{HCl}$ buffer ( $\mathrm{pH}$ 7.4) containing $10 \mathrm{mM}$ EDTA and $0.5 \%$ Triton $\mathrm{X}-100$. After centrifugation for $5 \mathrm{~min}$ at $15,000 \mathrm{rpm}$, supernatant samples were treated with RNase A and proteinase $\mathrm{K}$. Subsequently, $20 \mu \mathrm{l}$ of $5 \mathrm{M} \mathrm{NaCl}$ and $120 \mu \mathrm{l}$ isopropanol were added to the samples, which were then kept at $-20^{\circ} \mathrm{C}$ for $6 \mathrm{~h}$. Then, following centrifugation for $15 \mathrm{~min}$ at $15,000 \mathrm{rpm}$, DNA pellets were dissolved in $20 \mu \mathrm{l}$ of TE buffer (10 mM Tris-HCl and $1 \mathrm{mM}$ EDTA) as loading samples. To assay the DNA fragmentation pattern, samples were loaded onto $1.5 \%$ agarose gel and electrophoresis was carried out.

DAPI staining. Cells were washed with cold PBS and fixed with $4 \%$ paraformaldehyde (Sigma-Aldrich Chemical Co.) in PBS for $10 \mathrm{~min}$ at room temperature. The fixed cells were washed with PBS and stained with DAPI solution for $10 \mathrm{~min}$ at room temperature. The cells were then washed twice with PBS and analyzed with a fluorescence microscope (Carl Zeiss, Germany).

Determination of caspase activity. The activities of caspases were determined by colorimetric assay kits, which utilize synthetic tetrapeptides [Asp-Glu-Val-Asp (DEAD) for caspase-3; Ile-Glu-Thr-Asp (IETD) for caspase-8; and Leu-Glu-His-Asp (LEHD) for caspase-9] labeled with p-nitroaniline (pNA), according to the manufacturer's protocol. The cells were briefly lysed in the supplied lysis buffer. The supernatants were collected and incubated with 


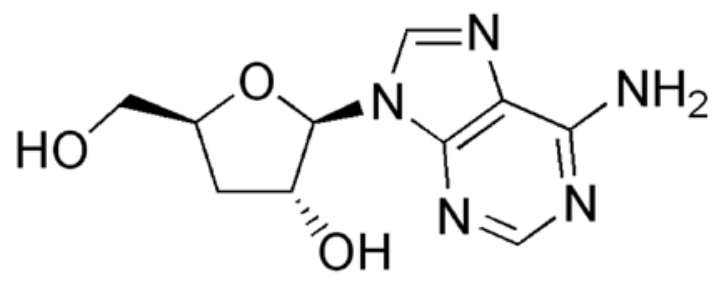

Figure 1. The chemical structure of cordycepin.

the supplied reaction buffer containing dithiothreitol (DTT) and substrates at $37^{\circ} \mathrm{C}$ for $2 \mathrm{~h}$ in the dark. The caspase activity was determined by measuring changes in absorbance at $405 \mathrm{~nm}$ using the ELISA reader.

Isolation of total-RNA and reverse transcription-PCR. Total-RNA was isolated using TRIzol reagent (Invitrogen, Carlsbad, CA). Total-RNA (1.0 $\mu \mathrm{g})$ obtained from cells was primed with random hexamers to synthesize complementary DNA using M-MLV reverse transcriptase (Promega, Madison, WI) according to the manufacturer's instructions. Single stranded cDNA was amplified by polymerase chain reaction (PCR) with the indicated primers. Amplification products obtained by PCR were electrophoretically separated on $1 \%$ agarose gel and visualized by ethidium bromide $(\mathrm{EtBr}$, Sigma-Aldrich Chemical Co.) staining.

Protein extraction and western blot analysis. The cells were harvested and lysed with lysis buffer $(20 \mathrm{mM}$ sucrose, $1 \mathrm{mM}$ EDTA, $20 \mu \mathrm{M}$ Tris-Cl,pH 7.2, 1 mM DTT, $10 \mathrm{mM} \mathrm{KCl}, 1.5 \mathrm{mM}$ $\mathrm{MgCl}_{2}$ and $5 \mu \mathrm{g} / \mathrm{ml}$ aprotinin) for $30 \mathrm{~min}$. The protein concentration was measured using a Bio-Rad protein assay (Bio-Rad Laboratories, Hercules, CA) according to the manufacturer's instructions. In a parallel experiment, cells were washed with cold PBS and scraped; cytoplasmic and nuclear proteins were then extracted using a mitochondrial fractionation kit according to the manufacturer's instructions (Activemotif, Carlsbad, CA). For western blot analysis, an equal amount of protein was subjected to electrophoresis on SDS-polyacrylamide gel and transferred by electroblotting to a nitrocellulose membrane (Schleicher \& Schuell, Keene, NH). The blots were probed with the desired antibodies for $1 \mathrm{~h}$, incubated with the diluted enzyme-linked secondary antibody and visualized by ECL kit according to the recommended procedure.

Mitochondrial membrane potential (MMP, $\Delta \Psi m)$ assay. The MMP of intact cells was measured by DNA flow cytometry with the lipophilic cation JC-1. JC-1 is a ratiometric, dual-emission fluorescent dye that is internalized and concentrated by respiring mitochondria; therefore, it can reflect changes in MMP in living cells. There are two excitation wavelengths: at low values of MMP, it remains a monomer (FL-1, green fluorescence; $527 \mathrm{~nm}$ ) while it forms aggregates at high MMP (FL-2, red fluorescence; $590 \mathrm{~nm}$ ), according to the recommended procedure (Calbiochem). For this study, the cells were trypsinized and the cell pellets were resuspended in PBS and incubated with $10 \mu \mathrm{M} \mathrm{JC}-1$ for $20 \mathrm{~min}$ at $37^{\circ} \mathrm{C}$. The cells were subsequently washed once with cold PBS, suspended and analyzed using a flow cytometer.
Measurement of intracellular ROS generation. The generation of ROS was determined in cells treated with cordycepin in the presence and absence of NAC, and was evaluated with 5-(and 6)-carboxy-2'7'-dichlorodihydrofluorescein diacetate (DCF-DA; Molecular Probes, Leiden, The Netherlands) as described previously (27). The cells were incubated with $10 \mu \mathrm{M}$ DCF-DA at $37^{\circ} \mathrm{C}$ for $30 \mathrm{~min}$. The cells were then washed with PBS and FL-1 fluorescence was measured with a flow cytometer.

Statistical analysis. The data are expressed as a mean \pm SD. A statistical comparison was performed using one-way ANOVA followed by a Fisher's test. The significant $(\mathrm{p}<0.05)$ differences between the groups were determined using an unpaired Student's t-test.

\section{Results}

Induction of apoptosis by cordycepin in PC-3 cells. To investigate the effect of cordycepin on cell growth of human prostate carcinoma cell lines (PC-3, LNCaP and DU145), the cells were exposed to various concentrations $(0,5,10,15,20$ and $25 \mu \mathrm{g} / \mathrm{ml})$ of cordycepin for $24 \mathrm{~h}$ and then cell viability was measured by the MTT assay. As shown in Fig. 2A, cordycepin elicited a decrease in cell viability in a dose-dependent manner in prostate carcinoma cells; notably, the cytotoxicity of cordycepin was more potent in PC-3 cells than in DU145 and LNCaP cells. Morphological analysis of DAPI-stained PC-3 cells treated with cordycepin indicated that they had undergone gross morphological changes indicative of apoptosis, including cell shrinkage, chromatin condensation and the loss of nuclear construction (Fig. 2B). Further experiments were performed to determine if this inhibitory effect of cordycepin on cell viability was the result of apoptotic cell death. As shown in Fig. 2C, cordycepin treatment resulted in an increased accumulation of PC-3 cells at the apoptotic sub-G1 phase of the cell cycle and that this response occurred in a concentration-dependent manner. We also examined whether or not cordycepin induces DNA fragmentation, another hallmark of apoptosis. Following agarose gel electrophoresis of cells treated with cordycepin, a typical ladder pattern of inter-nucleosomal DNA fragmentation was observed (Fig. 2D). These results clearly suggest that cordycepin-induced apoptosis took place in PC-3 cells.

Activation of caspase-3 and -9 by cordycepin in PC-3 cells. Caspases are a family of cysteine proteases that play essential roles as important mediators in apoptosis and as determinants of the general apoptotic morphology through the cleavage of various cellular substrates including PARP, an endogenous substrate of activated caspase-3 (28). Caspase-3, a pivotal mediator of apoptosis in mammalian cells, can be activated by upstream initiator caspases such as caspase- 8 or -9 through two distinct pathways (i.e., the death receptor-mediated extrinsic caspase-8 pathway or the mitochondria dependent-cytochrome $c /$ caspase-9 intrinsic pathway, respectively) $(8,9)$. Therefore, we investigated whether cordycepin induces the activation of caspases and cleavage of PARP in PC-3 cells. As shown in Fig. 3A, western blot analyses showed that cordycepin concentration-dependently induced a marked decrease of pro-caspase-3 and -9, and cleavage of PARP. In addition, to quantify the proteolytic activation of the caspases, we evaluated 
A

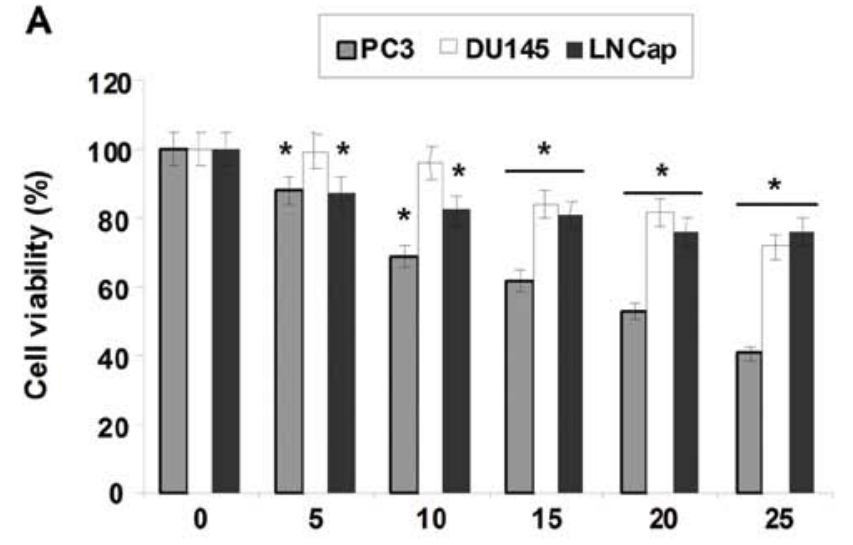

Cordycepin $(\mu \mathrm{g} / \mathrm{ml})$
B

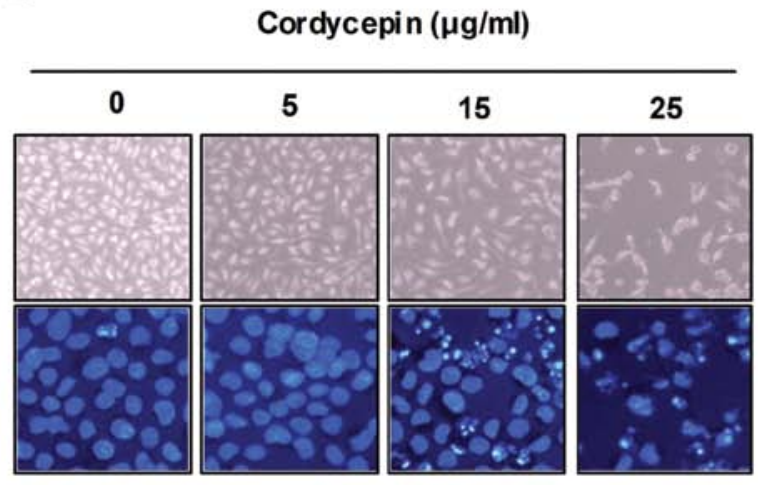

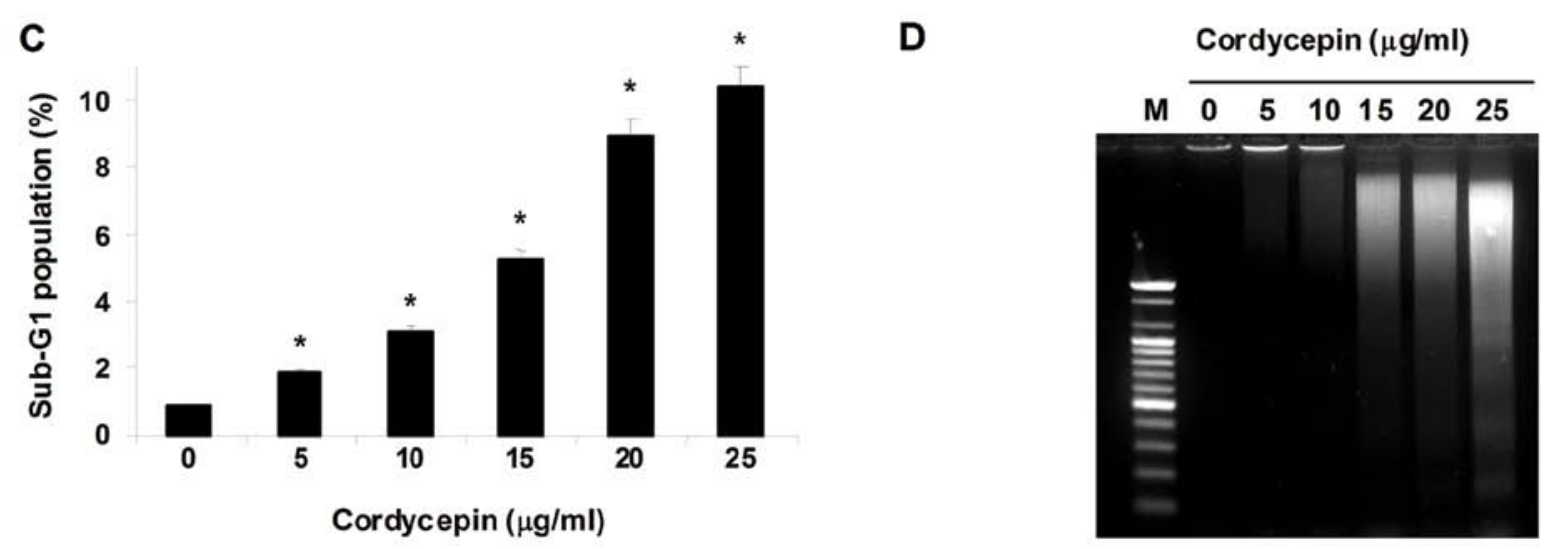

Figure 2. Inhibition of cell viability and induction of apoptosis by cordycepin in human prostate cancer cells. (A) PC-3, DU145 and LNCaP cells ( $2 \times 10^{5}$ cells/well) were plated in 6-well tissue culture plates; next, the cells were treated with variable concentrations of cordycepin for 24 h. Following treatment, the cell viability was determined by MTT assays. Data are the mean \pm SD of three different experiments. The significance was determined by a Student's t-test ( ${ }^{*}<0.05$, compared with control). (B) The cellular (upper panels) and nuclear (lower panels) morphological changes of PC-3 cells incubated with or without cordycepin for $24 \mathrm{~h}$ were examined under an inverted microscope (magnification, x200) and a fluorescence microscope (x400), respectively. For DAPI staining (lower panels), the cells were fixed and stained with DAPI solution for $10 \mathrm{~min}$ at room temperature. (C) To quantify the degree of apoptosis induced by cordycepin, cells grown under the same conditions as (A) were evaluated by a flow cytometer for sub-G1 DNA content, which represents the cells undergoing apoptotic DNA degradation. Data are the mean \pm SD of three different experiments. The significance was determined by the Student's t-test $\left({ }^{*} \mathrm{p}<0.05\right.$, compared with control). (D) To analyze the DNA fragmentation, the cells were treated with the indicated concentrations of cordycepin for $24 \mathrm{~h}$; DNA was extracted, resolved in $1.5 \%$ agarose gel and then visualized using EtBr. The results presented here are from one representative experiment of three performed that showed similar patterns.

A

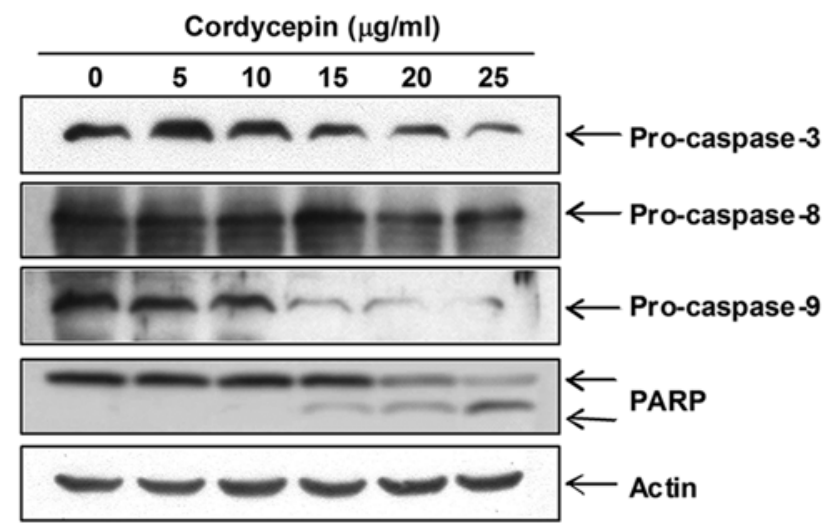

B

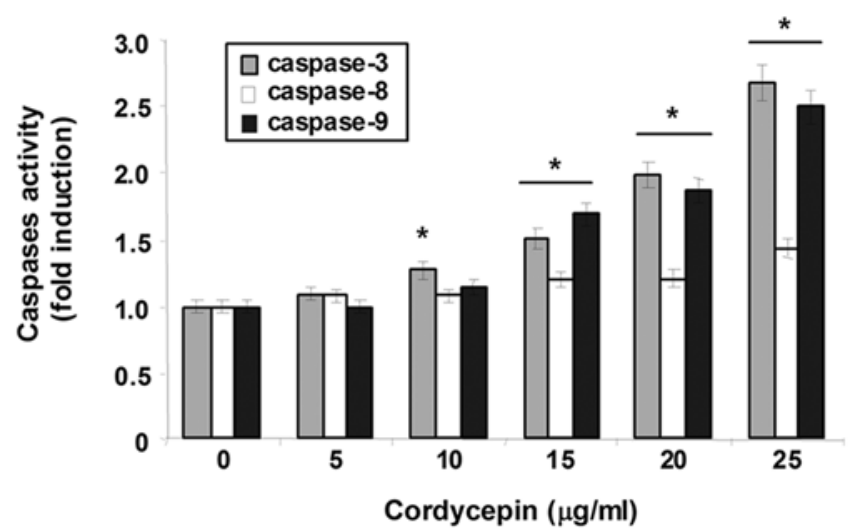

Figure 3. Effects of cordycepin on the expression and activity of caspases in PC-3 cells. (A) The cells were treated with the indicated concentrations of cordycepin for $24 \mathrm{~h}$. Equal amounts of cell lysates $(40 \mu \mathrm{g})$ were then resolved by SDS-polyacrylamide gels, transferred to nitrocellulose membranes and probed with antibodies against caspase-3,-8 and -9 and PARP. The proteins were then visualized using ECL detection. Actin was used as an internal control. (B) The cell lysates obtained from cells grown under the same conditions as (A) were assayed for in vitro caspase-3, -8 and -9 activity using DEVD-pNA, IETD-pNA and LEHD-pNA, respectively, as substrates. The relative fluorescent products were measured. Data are means \pm SD from representative experiments performed at least three times. The significance was determined by the Student's t-test $\left({ }^{*} \mathrm{p}<0.05\right.$, compared with control). 

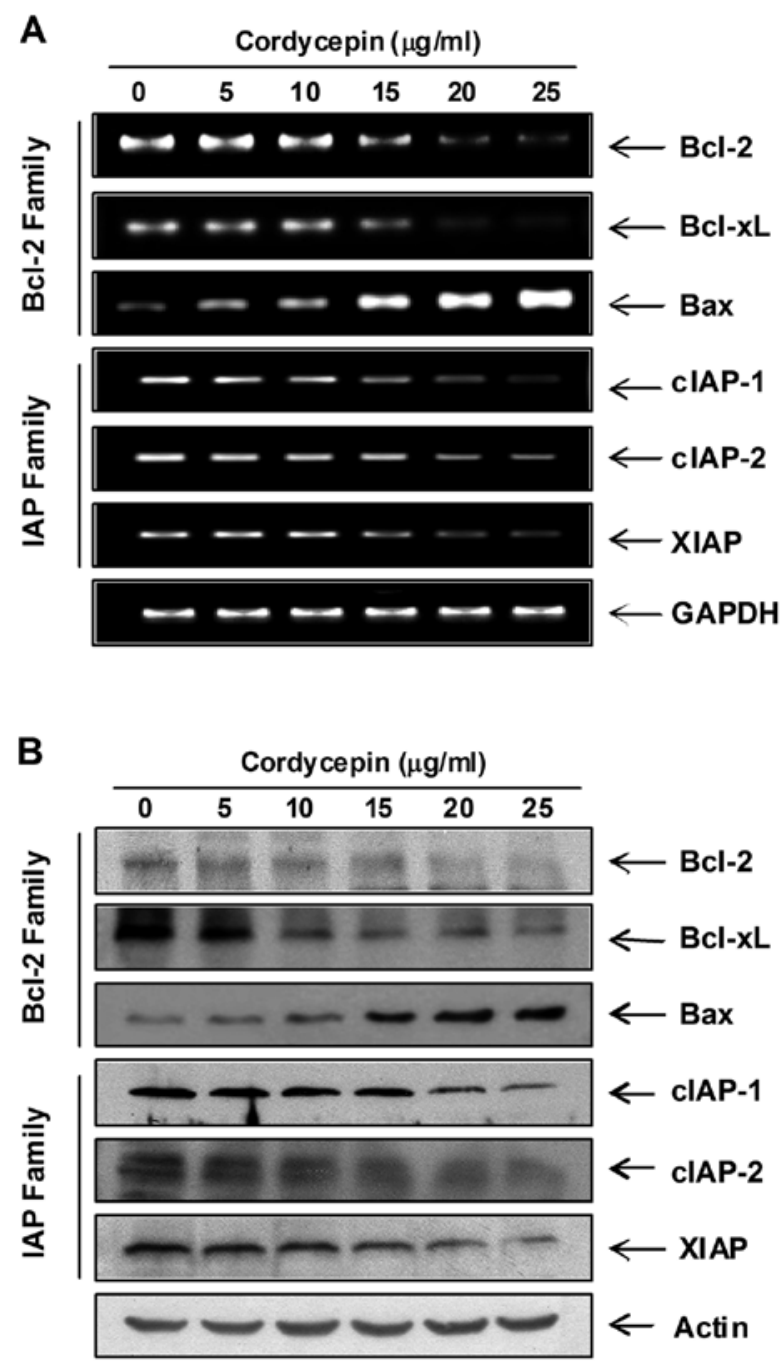

Figure 4. Effects of cordycepin on the levels of Bcl-2 and IAP family members in PC-3 cells. (A) The cells were treated with the indicated concentrations of cordycepin for $24 \mathrm{~h}$. Proteins on western blots were detected with the indicated antibodies and ECL detection. Actin was used as an internal control. (B) After 24-h incubation with cordycepin, total-RNAs were isolated and reverse-transcribed. The resulting cDNAs were subjected to PCR with the indicated primers, and the reaction products were subjected to electrophoresis in $1 \%$ agarose gel and visualized by EtBr staining. GAPDH was used as an internal control.

in vitro caspase activities using fluorogenic substrates. As shown in Fig. 3B, treatment with cordycepin significantly increased the activities of caspase- 3 and -9 compared with control cells, though it did not affect that of caspase-8, suggesting a likely involvement of mitochondria-dependent cascade for caspase activation.

Modulation of Bcl-2 and IAP family and dysfunction of mitochondria by cordycepin in PC-3 cells. Next, we examined the effect of cordycepin on the expression of Bcl-2 and IAP family members, which have been reported to play an important role in regulating apoptosis. RT-PCR and western blot analysis data showed that cordycepin concentration-dependently induced the expression of pro-apoptotic Bax mRNA and protein, whereas those levels of anti-apoptotic Bcl-2 and Bcl-xL were decreased in response to cordycepin treatment. The levels of IAP family members such as XIAP, cIAP-1 and cIAP-2 were markedly
A
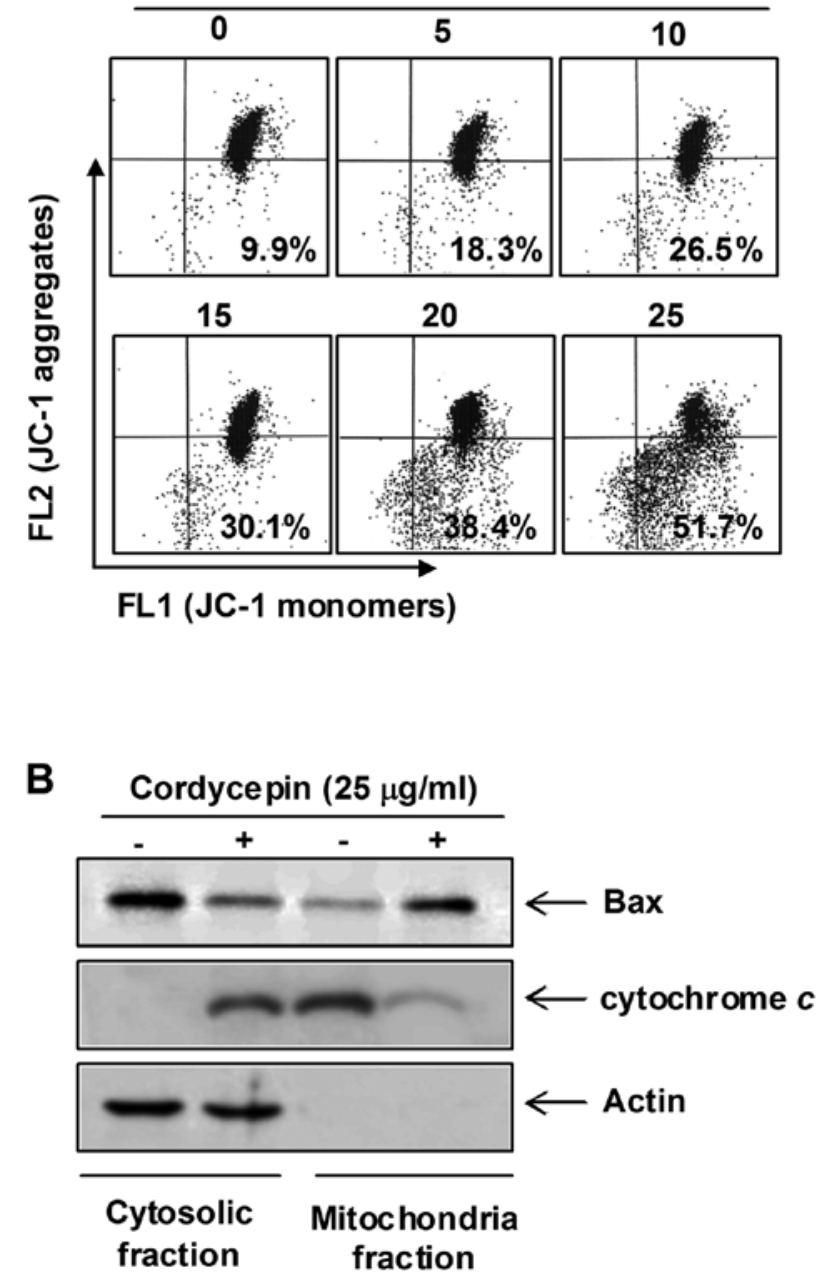

Figure 5. Effects of cordycepin treatment on the levels of mitochondrial membrane hyperpolarization and cytochrome c in PC-3 cells. (A) The cells were incubated with various concentrations of cordycepin for $24 \mathrm{~h}$, and stained with JC-1 for $20 \mathrm{~min}$ at $37^{\circ} \mathrm{C}$. The mean JC-1 fluorescence intensity was then detected using a flow cytometer. Data represent the means of two independent experiments. (B) Cytosolic and mitochondrial extracts were prepared from control and cordycepin-treated cells, resolved by SDS-polyacrylamide gels, transferred to nitrocellulose membranes, and probed with the anti-Bax and anti-cytochrome $c$ antibodies. Equal protein loading was evaluated by comparison to an actin control.

inhibited by cordycepin treatment in a dose-dependent manner (Fig. 4). In order to assess the role of the mitochondria in cordycepin-induced apoptosis of PC-3 cells, we investigated the effects of cordycepin on the levels of cytosolic and mitochondrial Bax and cytochrome $c$ as well as the MMP values.

As shown in Fig. 5A, treatment with cordycepin concentration-dependently caused a significant reduction in the MMP value in PC-3 cells. Furthermore, exposure of cells to cordycepin led to a significant increase in the release of the mitochondrial pro-apoptotic protein cytochrome $c$ to the cytosol and a decreased Bax level of cytosol (Fig. 5B). In contrast, treatment with cordycepin induced a significant decrease in mitochondrial cytochrome $c$ and an increase of Bax protein into the mitochondria, indicating a direct role of the mitochondria in cordycepin-induced apoptosis of PC-3 cells. 
A

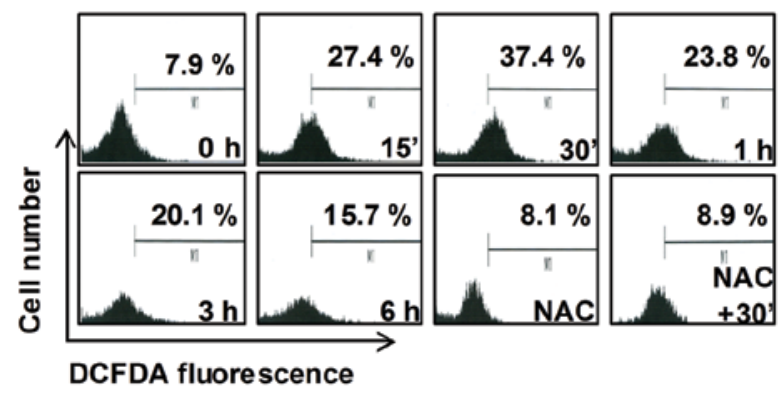

B

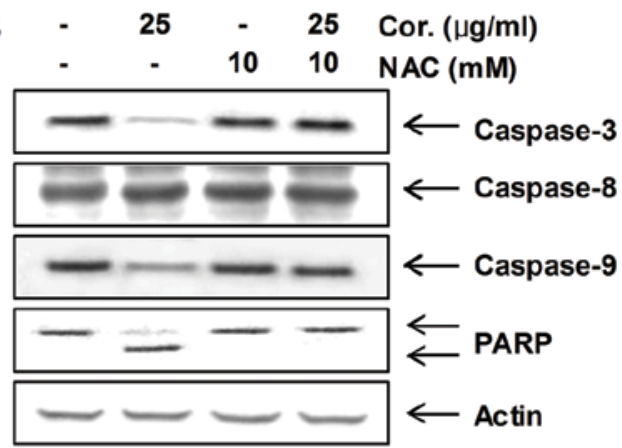

C
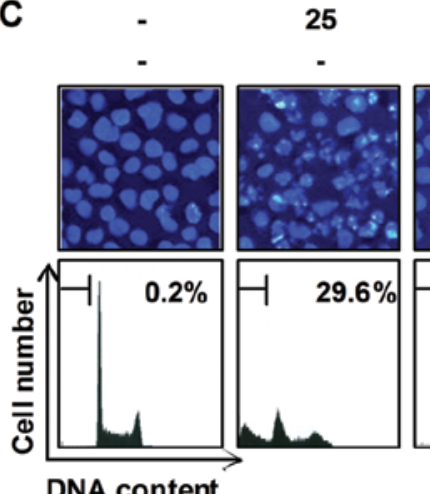

DNA content
10
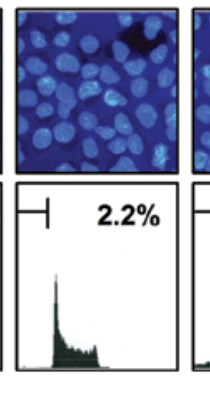

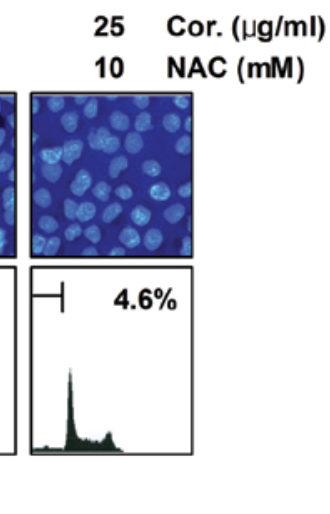

25

Cor. $(\mu \mathrm{g} / \mathrm{ml})$ NAC (mM)

Figure 6. Cordycepin-induced apoptosis is associated with ROS generation in PC-3 cells. (A) Cells were treated with or without NAC (10 mM) for 1 h before being challenged with $30 \mu \mathrm{g} / \mathrm{ml}$ of cordycepin for the indicated times. ROS generation was measured by a flow cytometer. (B) Cells were treated with or without NAC for $1 \mathrm{~h}$ before being challenged with $30 \mu \mathrm{g} / \mathrm{ml}$ of cordycepin for $30 \mathrm{~min}$. Equal amounts of cell lysates (40 $\mu \mathrm{g}$ ) were then resolved by SDS-polyacrylamide gels, transferred to nitrocellulose membranes, and probed with antibodies against caspase-3, -8 , -9 and PARP. The proteins were then visualized using ECL detection. Actin was used as an internal control. (C) The nuclear morphological changes in PC-3 cells incubated under the same conditions as (B) were analyzed via fluorescence microscope (magnification, x400). For DAPI staining, the cells were fixed and stained with DAPI solution for 10 min at room temperature (upper panels). To quantify the degree of apoptosis induced by cordycepin, cells grown under the same conditions as (B) were evaluated by a flow cytometer for sub-G1 DNA content. Each point represents the average of two independent experiments (lower panels). (D) The cell lysates obtained from cells grown under the same conditions as (B) were assayed for in vitro caspase-3, -8 and -9 activity using DEVD-pNA, IETD-pNA and LEHD-pNA, respectively, as substrates. The relative fluorescent products were measured. Data are means \pm SD from representative experiments performed at least three times. The significance was determined by a Student's t-test ( ${ }^{*} \mathrm{p}<0.05$, compared with control).

Involvement ROS generation in cordycepin-induced apoptosis in PC-3 cells. Many reports have suggested that the mitochondrial apoptotic pathway is an important downstream signal of ROS in apoptotic cell death. High levels of ROS can induce apoptosis by triggering mitochondrial permeability transition pore opening, release of pro-apoptotic factors and activation of caspase- 9 and $-3(12,13)$. Thus, to examine whether the ROS accumulation is involved in cordycepin-induced apoptosis, intracellular ROS levels were examined using DCFH-DA and flow cytometry. As shown in Fig. 6A, treatment of PC-3 cells with cordycepin resulted in a significant elevation of intracellular ROS, compared with the vehicle control. In a parallel experiment, pre-treatment of the ROS scavenger NAC, along with cordycepin, significantly reduced ROS generation as compared to the cordycepin-treated group, whereas treatment with NAC alone did not alter ROS level in comparison to control. Furthermore, blocking of the generation of ROS by pre-treatment of the cells with NAC prevented the cordycepin-induced increased accumulation of sub-G1 population, activation of caspases (-3 and -9$)$, and proteolytic cleavage of PARP (Fig. 6B-D). In addition, NAC blocked modulation of Bcl-2 and IAP family proteins, loss of MMP, and translocation of cytochrome $c$ and Bax (Fig. 7). Taken together, the above findings suggest that cordycepin induces apoptosis via ROS generation-dependent mechanisms in PC-3 cells.

\section{Discussion}

Targeting apoptosis pathways is considered an effective strategy for cancer chemoprevention as well as therapy. Many chemopreventive agents have been found to modulate key molecules or events in apoptosis-inducing signal transduction pathways. In the present study, we evaluated the mechanisms by which cordycepin induced apoptotic cell death in PC-3 human prostate cancer cells through the generation of ROS. Our study demonstrated that treatment of cordycepin mediated mitochondria membrane dysfunction, resulting in the release of apoptotic genes, such as cytochrome $c$, from mitochondria into the cytosol. Ultimately, these results activate a caspase cascade and apoptotic cell death. Suppression of ROS generation attenuated this cordycepin-induced activation of caspase and subsequent apoptosis.

The process of apoptosis is controlled by a wide range of cellular signals, which can be divided into both extrinsic and intrinsic pathways. Apoptosis requires caspases activity, and 

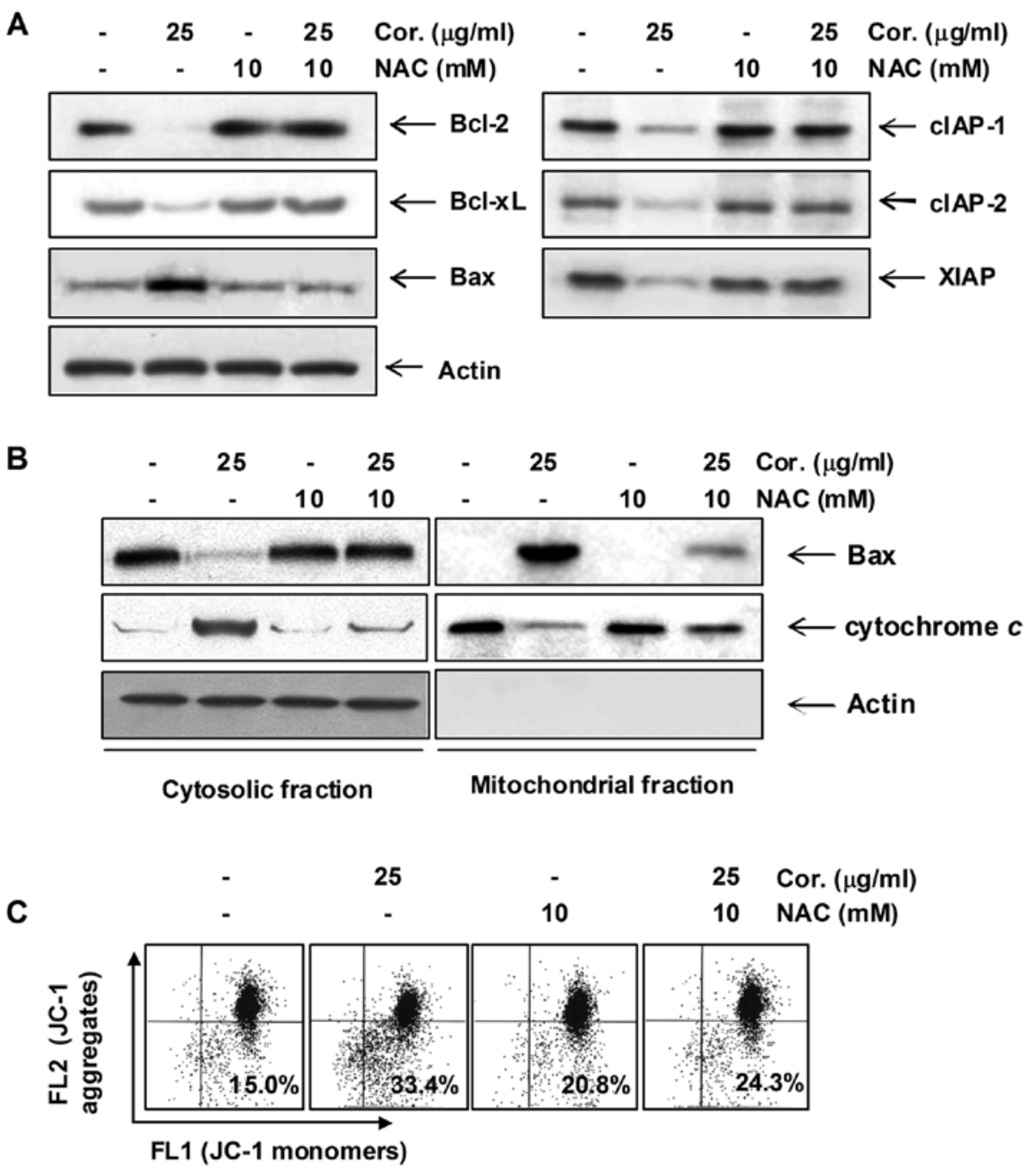

Figure 7. Inhibition of cordycepin-induced modulation of Bcl-2 and IAP family proteins and MMP values by blocking of the generation of ROS in PC-3 cells. (A) Cells were treated with or without NAC for $1 \mathrm{~h}$ before being challenged with $30 \mu \mathrm{g} / \mathrm{ml}$ of cordycepin for 30 min. Then, cells were separated by SDS-polyacrylamide gels and transferred onto nitrocellulose membranes. The membranes were probed with the indicated antibodies. Proteins were visualized using an ECL detection system. Actin was used as an internal control. (B) Cytosolic and mitochondrial extracts were prepared from cells grown under the same conditions, resolved by SDS-polyacrylamide gels, transferred to nitrocellulose membranes, and probed with the anti-Bax and anti-cytochrome $c$ antibodies. Equal protein loading was evaluated by comparison to an actin control. (C) The cells were stained with JC-1 for $20 \mathrm{~min}$ at $37^{\circ} \mathrm{C}$. The mean JC-1 fluorescence intensity was then detected using flow cytometry. Data represent the means of two independent experiments.

caspases become active when cleaved $(29,30)$. Adaptor proteins facilitate the auto-cleavage of initiator caspases (e.g., caspase- 8 and -9), initiator caspases cleave effector caspases (e.g., caspase3), and effector caspases disrupt cell function to elicit cell death. Two events signal adaptor-mediated caspase cleavage: the binding of ligand to death receptors (the death receptor pathway) and the release of cytochrome $c$ from mitochondria (the mitochondrial pathway) $(8,9)$. Death receptors activate caspase- 8 , whereas cytochrome $c$ activates caspase-9. Caspase-3 is common to both pathways. When apoptosis occurs, many proteins modulate apoptotic signaling, including the IAPs and the Bcl-2 proteins. IAPs inactivate cleaved caspases; thus, they impede the apoptotic process once it has begun. Caspases targeted by IAPs include caspase- 9 and -3 but not caspase- 8 (31). The Bcl-2 proteins damage or protect mitochondria; types of Bcl-2 proteins are: multidomain apoptotic (Bax and Bak), single domain apoptotic (termed BH3-only), and anti-apoptotic (Bcl-2 and Bcl-xL) (32). When receiving an apoptosis signal, Bax and Bak perforate mitochondrial membranes to release cytochrome $c$. BH3-only proteins facilitate activation of Bax and Bak, whereas antiapoptotic Bcl-2 proteins oppose activation. Bak is constitutively mitochondrial, whereas Bax translocates from the cytosol to the mitochondria in response to stress $(33,34)$. Our results showed that cordycepin induced apoptosis in PC-3 cells, and this apoptosis was associated with increased activity of intrinsic caspase cascades, such as caspase-9 (Fig. 3). Treatment of cordycepin also reduced the expression of IAP family proteins, such as XIAP, cIAP-1 and cIAP-2, and the anti-apoptotic Bcl-2 and Bcl-xL, whereas the expression of pro-apoptotic Bax was markedly raised in PC-3 cells (Fig. 4). Additionally, cordycepin mediated the loss of MMP and release of cytochrome $c$ to cytosol, consistent with mitochondria-dependent apoptosis (Fig. 5), which was connected with the activation of caspase-9 and -3 , and the concomitant degradation of PARP (Fig. 2). Therefore, the apoptotic effects of cordycepin on PC-3 cells appeared to involve activation of the mitochondrial pathways. 
Additionally, ROS are known to mediate other intracellular signaling cascades, such as mitochondrial apoptosis (35). Oxidative stress is generally considered an important regulator of apoptosis (36). Many studies have suggested that a disproportionate production of ROS in mitochondria leads to oxidative stress and dysfunction of cell organelles like the mitochondria. This is associated with the release of mitochondrial factors, triggering caspase cascade and eventually apoptosis or necrosis $(12,13)$. We found a significant overproduction of ROS in cordycepin-treated PC-3 cells; however, treatment of cordycepin with NAC, a commonly used ROS scavenger, effectively blocked this ROS generation and almost completely suppressed the cordycepin-induced activation of caspases, degradation of PARP, decrease of sub-G1 population, modulation of $\mathrm{Bcl}-2$ as well as IAP family proteins, loss of MMP and release of cytochrome $c$ from mitochondria to cytosol (Figs. 6 and 7). Because ROS have the potential to induce the collapse of the MMP, and consequently trigger the series of events leading to the mitochondria-associated apoptotic pathway $(36,37)$, our findings suggest the involvement of ROS and mitochondrial dysfunction in cordycepin-induced caspase-mediated apoptosis in PC-3 cells.

In conclusion, the present study demonstrated that cordycepin could significantly induce apoptosis in human prostate PC-3 cells through a mitochondria-mediated caspase-dependent pathway. The positive correlation between the overproduction of ROS and mitochondrial dysfunction, together with the protective effect of NAC on the cordycepininduced apoptosis, suggest that ROS generation may play a key role in the apoptotic process induced by cordycepin. Taken together, these results suggest that cordycepin may be a potential chemotherapeutic agent for the treatment of prostate cancer patients.

\section{Acknowledgements}

This research was supported by Technology Development Program for Agriculture and Forestry (610003-03-1-SU000), Ministry for Food, Agriculture, Forestry and Fisheries, and Basic Science Research Program through the National Research Foundation of Korea (NRF) grant funded by the Korea government (no. 2012046358).

\section{References}

1. Jemal A, Ward E and Thun M: Declining death rates reflect progress against cancer. PLoS One 5: e9584-e9591, 2010.

2. Sciarra A, Salciccia S and Panebianco V: Proton spectroscopic and dynamic contrast-enhanced magnetic resonance: a modern approach in prostate cancer imaging. Eur Urol 54: 485-488, 2008.

3. Crawford ED: Epidemiology of prostate cancer. Urology 62: $3-12,2003$

4. Zhang L, Yang BX, Zhang HT, Wang JG, Wang HL and Zhao XJ: Prostate cancer: an emerging threat to the health of aging men in Asia. Asian J Androl 13: 574-578, 2011.

5. Xia SJ, Cui D and Jiang Q: An overview of prostate diseases and their characteristics specific to Asian men. Asian J Androl 14: 458-464, 2012.

6. Neto CC, Amoroso JW and Liberty AM: Anticancer activities of cranberry phytochemicals: an update. Mol Nutr Food Res 52: S18-S27, 2008.

7. Kaur M, Pop M, Shi D, Brignone C and Grossman SR: hHR23B is required for genotoxic-specific activation of p53 and apoptosis. Oncogene 26: 1231-1237, 2007.
8. Igney FH and Krammer PH: Immune escape of tumors: apoptosis resistance and tumor counterattack. J Leukoc Biol 71: 907-920, 2002.

9. $\mathrm{Hu} \mathrm{W}$ and Kavanagh JJ: Anticancer therapy targeting the apoptotic pathway. Lancet Oncol 4: 721-729, 2003.

10. Pathak N and Khandelwal S: Role of oxidative stress and apoptosis in cadmium induced thymic atrophy and splenomegaly in mice. Toxicol Lett 169: 95-108, 2007.

11. Chatterjee S, Kundu S, Bhattacharyya A, Hartinger CG and Dyson PJ: The ruthenium(II)-arene compound RAPTA-C induces apoptosis in EAC cells through mitochondrial and p53-JNK pathways. J Biol Inorg Chem 13: 1149-1155, 2008.

12. Huppertz B, Kadyrov M and Kingdom JC: Apoptosis and its role in the trophoblast. Am J Obstet Gynecol 195: 29-39, 2006.

13. Zhou H, Liu X, Liu L, Yang Z, Zhang S, Tang M, Tang Y, Dong $\mathrm{Q}$ and $\mathrm{Hu} \mathrm{R}$ : Oxidative stress and apoptosis of human brain microvascular endothelial cells induced by free fatty acids. J Int Med Res 37: 1897-1903, 2009.

14. Wang MF, Liao YF, Hung YC, Lin CL, Hour TC, Lue KH, Hung HC and Liu GY: Hydroxydibenzoylmethane induces apoptosis through repressing ornithine decarboxylase in human promyelocytic leukemia HL-60 cells. Exp Mol Med 43: 189-196, 2011.

15. Cunningham KG, Manson W, Spring FS and Hutchinson SA: Cordycepin, a metabolic product isolated from cultures of Cordyceps militaris. Nature 166: 949, 1950.

16. Paterson RR: Cordyceps: a traditional Chinese medicine and another fungal therapeutic biofactory? Phytochemistry 69: 1469-1495, 2008.

17. Horowitz B, Goldfinger BA and Marmur J: Effect of cordycepin triphosphate on the nuclear DNA-dependent RNA polymerases and poly(A) polymerase from the yeast, Saccharomyces cerevisiae. Arch Biochem Biophys 172: 143-148, 1976.

18. Müller WE, Seibert G, Beyer R, Breter HJ, Maidhof A and Zahn RK: Effect of cordycepin on nucleic acid metabolism in L5178Y cells and on nucleic acid-synthesizing enzyme systems. Cancer Res 37: 3824-3833, 1977.

19. Foss FM: Combination therapy with purine nucleoside analogs. Oncology 14: 31-35, 2000.

20. Nakamura K, Yoshikawa N, Yamaguchi Y, Kagota S, Shinozuka K and Kunitomo M: Antitumor effect of cordycepin (3'-deoxyadenosine) on mouse melanoma and lung carcinoma cells involves adenosine A3 receptor stimulation. Anticancer Res 26: 43-47, 2006.

21. Sugar AM and McCaffrey RP: Antifungal activity of 3'-deoxyadenosine (cordycepin). Antimicrob Agents Chemother 42: 1424-1427, 1998.

22. Ahn YJ, Park SJ, Lee SG, Shin SC and Choi DH: Cordycepin: selective growth inhibitor derived from liquid culture of Cordyceps militaris against Clostridium spp. J Agric Food Chem 48: 2744-2748, 2000.

23. Kim HG, Shrestha B, Lim SY, Yoon DH, Chang WC, Shin DJ, Han SK, Park SM, Park JH, Park HI, Sung JM, Jang Y, Chung N, Hwang KC and Kim TW: Cordycepin inhibits lipopolysaccharide-induced inflammation by the suppression of NF-kappaB through Akt and p38 inhibition in RAW 264.7 macrophage cells. Eur J Pharmacol 545: 192-199, 2006.

24. Jeong JW, Jin CY, Kim GY, Lee JD, Park C, Kim GD, Kim WJ, Jung WK, Seo SK, Choi IW and Choi YH: Anti-inflammatory effects of cordycepin via suppression of inflammatory mediators in BV2 microglial cells. Int Immunopharmacol 10: 1580-1586, 2010.

25. Zhou X, Gong Z, Su Y, Lin J and Tang K: Cordyceps fungi: natural products, pharmacological functions and developmental products. J Pharm Pharmacol 61: 279-291, 2009.

26. Lee K, Lee MH, Kang YW, Rhee KJ, Kim TU and Kim YS: Parkin induces apoptotic cell death in TNF- $\alpha$-treated cervical cancer cells. BMB Rep 45: 526-531, 2012.

27. Kim IH, Kim SW, Kim SH, Lee SO, Lee ST, Kim DG, Lee MJ and Park WH: Parthenolide-induced apoptosis of hepatic stellate cells and anti-fibrotic effects in an in vivo rat model. Exp Mol Med 44: 448-456, 2012.

28. Wang X, Chen S, Ma G, Ye M and Lu G: Involvement of proinflammatory factors, apoptosis, caspase -3 activation and $\mathrm{Ca}^{2+}$ disturbance in microglia activation-mediated dopaminergic cell degeneration. Mech Ageing Dev 126: 1241-1254, 2005.

29. Chang HY and Yang X: Proteases for cell suicide: functions and regulation of caspases. Microbiol Mol Biol Rev 64: 821-846, 2000 .

30. Jin Z and El-Deiry WS: Overview of cell death signaling pathways. Cancer Biol Ther 4: 139-163, 2005. 
31. Deveraux QL, Roy N, Stennicke HR, Van Arsdale T, Zhou Q, Srinivasula SM, Alnemri ES, Salvesen GS and Reed JC: IAPs block apoptotic events induced by caspase-8 and cytochrome c by direct inhibition of distinct caspases. EMBO J 17: 2215-2223, 1998.

32. Borner C: The Bcl-2 protein family: sensors and checkpoints for life-or-death decisions. Mol Immunol 39: 615-647, 2003.

33. Griffiths GJ, Dubrez L, Morgan CP, Jones NA, Whitehouse J, Corfe BM, Dive C and Hickman JA: Cell damage-induced conformational changes of the pro-apoptotic protein Bak in vivo precede the onset of apoptosis. J Cell Biol 144: 903-914, 1999.

34. Wolter KG, Hsu YT, Smith CL, Nechushtan A, Xi XG and Youle RJ: Movement of Bax from the cytosol to mitochondria during apoptosis. J Cell Biol 139: 1281-1292, 1997.
35. Bruce-Keller AJ, Begley JG, Fu W, Butterfield DA, Bredesen DE, Hutchins JB, Hensley K and Mattson MP: Bcl-2 protects isolated plasma and mitochondrial membranes against lipid peroxidation induced by hydrogen peroxide and amyloid beta-peptide. J Neurochem 70: 31-39, 1998.

36. Fiers W, Beyaert R, Declercq W and Vandenabeele P: More than one way to die: apoptosis, necrosis and reactive oxygen damage. Oncogene 18: 7719-7730, 1999.

37. Fleury C, Mignotte B and Vayssière JL: Mitochondrial reactive oxygen species in cell death signaling. Biochimie 84: 131-141, 2002 . 